\title{
Development of In Vitro-In Vivo Correlation for Upadacitinib Extended-Release Tablet Formulation
}

\author{
Mohamed-Eslam F. Mohamed, ${ }^{1,3}$ Sheryl Trueman, ${ }^{1}$ Ahmed A. Othman, ${ }^{1}$ Jian-Hwa Han, ${ }^{2}$ \\ Tzuchi R. Ju, ${ }^{2}$ and Patrick Marroum ${ }^{1}$
}

Received 18 June 2019; accepted 30 August 2019; published online 25 October 2019

\begin{abstract}
Upadacitinib is a selective Janus Kinase 1 inhibitor which is being developed for the treatment of several inflammatory diseases including rheumatoid arthritis. Upadacitinib was evaluated in Phase 3 studies as an oral extended-release (ER) formulation administered once daily. The purpose of this study was to develop a level A in vitro-in vivo correlation (IVIVC) for upadacitinib ER formulation. The pharmacokinetics of four upadacitinib extended-release formulations with different in vitro release characteristics and an immediate-release capsule formulation of upadacitinib were evaluated in 20 healthy subjects in a single-dose, randomized, crossover study. In vivo pharmacokinetic data and in vitro dissolution data (USP Dissolution Apparatus 1; $\mathrm{pH} 6.8 ; 100 \mathrm{rpm}$ ) were used to establish a level A IVIVC. Three formulations were used to establish the IVIVC, and the fourth formulation was used for external validation. A non-linear IVIVC best described the relationship between upadacitinib in vitro dissolution and in vivo absorption profiles. The absolute percent prediction errors (\%PE) for upadacitinib $C_{\max }$ and AUC were less than $10 \%$ for all three formulations used to establish the IVIVC, as well as for the \% PE for the external validation formulation and the overall mean internal validation. Model was crossvalidated using the leave-one-out approach; all evaluated cross-validation runs met the regulatory acceptance criteria. A level A IVIVC was successfully developed and validated for upadacitinib ER formulation, which meets the FDA and EMA regulatory validation criteria and can be used as surrogate for in vivo bioequivalence.
\end{abstract}

KEY WORDS: upadacitinib; ABT-494; in vitro/in vivo correlations (IVIVC); extended-release formulation; pharmacokinetics.

\section{INTRODUCTION}

Upadacitinib (ABT-494) is a novel, selective Janus kinase (JAK) 1 inhibitor that potently inhibits JAK 1 , but is less potent against other JAK isoforms [1]. In addition to several normal physiological functions, JAKs play an essential role in the signaling of numerous cytokines involved in inflammatory disorders and inhibition of JAKs can provide approach for the treatment of patients with chronic systemic inflammatory diseases [2-4]. Upadacitinib is being developed for the treatment of several inflammatory diseases, including rheumatoid arthritis (RA), as the enhanced selectivity of

Electronic supplementary material The online version of this article (https://doi.org/10.1208/s12248-019-0378-y) contains supplementary material, which is available to authorized users.

\footnotetext{
${ }^{1}$ Clinical Pharmacology and Pharmacometrics, AbbVie Inc., 1 North Waukegan Road, AP31-3, North Chicago, Illinois 60064, USA.

${ }^{2}$ Dissolution Sciences, AbbVie Inc., North Chicago, Illinois, USA.

${ }^{3}$ To whom correspondence should be addressed. (e-mail: mohamedeslam.mohamed@abbvie.com)
}

upadacitinib against JAK 1 may offer an improved benefitrisk profile compared to less selective JAK inhibitors [5,6]. Upadacitinib recently demonstrated efficacy in five global Phase 3 trials in subjects with rheumatoid arthritis [7-10] and is currently under regulatory review by different global regulatory agencies for treatment of moderate-to-severe rheumatoid arthritis.

Upadacitinib pharmacokinetics was characterized in healthy subjects following the administration of the immediate-release (IR) and the extended-release (ER) formulations [11,12]. The extended-release (ER) tablet formulation of upadacitinib (the to-be-marketed formulation) was developed with the objective of decreasing the peak-totrough fluctuations in plasma concentrations with once-daily dosing. Under fasting conditions, peak plasma concentrations of upadacitinib were reached within $2 \mathrm{~h}$ of administration of the ER tablet [12]. Upadacitinib plasma exposures were doseproportional over the range of IR and ER doses evaluated in clinical studies; this encompassed doses ranging from 1 to $48 \mathrm{mg}$ using the IR formulation and 7.5 to $45 \mathrm{mg}$ using the ER formulation [11-14]. The bioavailability of the ER 
formulation used in Phase 3 studies was estimated to be $76 \%$ relative to the IR formulation [12,15]. Upadacitinib $15 \mathrm{mg}$ QD and $30 \mathrm{mg}$ QD using the ER formulation (the doses evaluated in Phase $3 \mathrm{RA}$ studies) provide equivalent daily AUC and comparable $C_{\max }$ and $C_{\min }$ to $6 \mathrm{mg}$ and $12 \mathrm{mg}$ BID using the IR formulation under fasting conditions [12]. Based on in vitro assessments, upadacitinib has been shown to be highly permeable and highly soluble at clinically relevant doses across the $\mathrm{pH}$ range of 1 to 7.5 (data on file at AbbVie). Based on in vitro assessments, upadacitinib is considered to be a class I drug according to the Biopharmaceutics Classification System [16]. The release-controlling polymer used in the upadacitinib ER formulation is hydroxypropyl methyl cellulose (HPMC), which forms a gel layer during dissolution and controls drug release through diffusion of the drug molecule and erosion of polymer chains.

Throughout the life-cycle of a drug product, the need often arises to change some aspects of the formulation, the manufacturing process, or the manufacturing site. Some postapproval changes require the need to demonstrate bioequivalence between the modified and the marketed formulation through an in vivo clinical study that is adequately powered to demonstrate bioequivalence $[17,18]$. Further, establishing in vivo-in vitro correlation (IVIVC) can allow for the prediction of the plasma concentration time profile of a formulation without having to conduct in vivo bioavailability studies, thus saving time and costs and avoiding the need to administer a drug to healthy volunteers [19]. The availability of a predictive IVIVC can facilitate the establishment of clinically meaningful dissolution specifications for release and reduce the number of in vivo bioavailability studies which may be needed to approve and maintain a drug product on the market $[17,18]$.

In the present study, the in vitro dissolution and in vivo pharmacokinetics for four ER formulations (30 mg strength) with various in vitro release profiles, including the proposed commercial formulation, were evaluated relative to a $24-\mathrm{mg}$ dose of the immediate-release (IR) capsule formulation. The methodology for the evaluation and establishment of a nonlinear level A IVIVC for upadacitinib ER formulations are described.

\section{MATERIALS AND METHODS}

\section{Formulations}

Four ER formulations (A, B, C, and D) were developed with identical amounts of upadacitinib $(30 \mathrm{mg})$ but varying amounts of the release-controlling polymer HPMC. Three formulations (Formulation A, Formulation B, and Formulation D) contained 10\%, 15\%, and 35\% HPMC, respectively. The fourth formulation evaluated (Formulation C) contained $20 \%$ HPMC as a prototype for the planned commercial formulation. A single 24-mg dose of upadacitinib $(2 \times 12 \mathrm{mg}$ IR upadacitinib capsules) was used as the reference formulation in the in vivo clinical study.

\section{In Vitro Dissolution Study}

A USP Dissolution Apparatus 1 operating at a rotating speed of $100 \mathrm{rpm}$ was used to generate the in vitro dissolution profiles. The dissolution medium was $900 \mathrm{~mL}$ of $0.05 \mathrm{M}$ phosphate buffer ( $\mathrm{pH}$ 6.8). An automatic sampler collected $1.5 \mathrm{~mL}$ of each of the sample at multiple time points $(0,1,2$, $4,6,8,10,12,16,20,24 \mathrm{~h})$, and the samples were then analyzed with high-performance liquid chromatography (HPLC). Several additional dissolution methods were also evaluated (Supplemental Table 1) in an attempt to mimic the deconvolved in vivo profiles and to establish a linear IVIVC (methods included different $\mathrm{pH}$ media, stirring speeds, dual$\mathrm{pH}$ conditions, dual-RPM conditions, and use of surfactants).

\section{In Vivo Bioavailability Study}

A Phase 1, single-dose, open-label study conducted according to a five-period, randomized, crossover design was used to characterize the bioavailability of four upadacitinib ER tablet formulations with different dissolution release rates relative to upadacitinib immediate-release capsules under fasting conditions. The study was conducted at PPD Development (Austin, TX, USA), and subjects were confined to the study site and supervised for approximately 21 consecutive days. Adult male subjects $(N=20)$ in general good health were selected to participate in the study. All procedures performed in studies involving human participants were in accordance with the ethical standards of the institutional and/ or national research committee (Salus IRB, Austin, TX) and with the 1964 Helsinki Declaration and its later amendments or comparable ethical standards. Informed consent was obtained from all individuals included in the study prior to any study-related procedure. Enrolled subjects were randomly assigned in equal numbers to one of five sequences of regimens $\mathrm{A}, \mathrm{B}, \mathrm{C}, \mathrm{D}$, and $\mathrm{E}$ consisting of five periods. In each of the five periods, a single dose of upadacitinib was taken orally with approximately $240 \mathrm{~mL}$ of water after a fasting for a minimum of $10 \mathrm{~h}$ and at least $4 \mathrm{~h}$ before lunch. Each dose was separated by a washout interval of 4 days. Blood samples for upadacitinib assay were collected into dipotassium ethylenediaminetetraacetic acid-containing collection tubes prior to dosing $(0 \mathrm{~h})$ and at $0.25,0.5,1,1.5,2,3,4,6,8,10$, $12,16,24,36,48$, and $72 \mathrm{~h}$ after dosing in each period. Plasma concentrations of upadacitinib were determined using a validated liquid chromatography method with tandem mass spectrometry [11]. The lower limit of quantitation (LLOQ) for upadacitinib was established at $0.05 \mathrm{ng} / \mathrm{mL}$; the assay coefficient of variation $(\% \mathrm{CV})$ was $\leq 8.9 \%$, and the mean absolute bias was $\leq 4.1 \%$.

\section{Upadacitinib Pharmacokinetic Parameters}

Pharmacokinetic parameters were estimated using noncompartmental methods in Phoenix ${ }^{\circledR}$ Version 7.0 (Pharsight, A Certara ${ }^{\circledR}$ Company, St. Louis, MO, USA). The maximum observed plasma concentration $\left(C_{\max }\right)$ of upadacitinib and time to $C_{\max }\left(t_{\max }\right)$ values were determined directly from the plasma concentration versus time data for each subject. The apparent terminal phase elimination rate constant ( $\beta$, BETA) was obtained from the slope of the least squares linear regression of the logarithms of the plasma concentration versus time data from the terminal log-linear phase of the profile. The terminal phase elimination half-life $\left(t_{1 / 2}\right)$ was calculated as $\ln (2) / \beta$. The area under the plasma 
concentration versus time curve from zero to the last measurable concentration $\left(\mathrm{AUC}_{\mathrm{t}}\right)$ and area under the plasma concentration versus time curve from zero to infinity $\left(\mathrm{AUC}_{\mathrm{inf}}\right)$ were calculated by the linear trapezoidal method.

\section{Development of In Vitro-In Vivo Correlation (IVIVC)}

A two-stage procedure was employed to develop a level A IVIVC: in vivo plasma concentration versus time profiles were first deconvolved followed by correlation of the fraction of drug absorbed and the fraction of drug dissolved. All numerical IVIVC analyses were conducted using IVIVC Toolkit ${ }^{\circledR}$ within Phoenix ${ }^{\circledR}$.

The following steps were followed to develop the IVIVC model:

1) The in vitro dissolution time profile for each of the four formulations were fitted to a Hill (Eq. 1) or Weibull functions (Eq. 2).

$F_{\text {diss,vitro }}(t)=\frac{F_{\text {inf }} * \mathrm{t}^{b}}{\mathrm{MDT}^{b}+t^{b}}$

where $F_{\text {diss, vitro }}$ is the fitted fraction dissolved at time $t, F_{\text {inf }}$ is the fraction dissolved at time infinity-fixed to 1 , MDT is the mean dissolution time (hours), and $b$ is the slope factor.

$F_{\text {diss,vitro }}(\mathrm{t})=F_{\text {inf }} *\left(1-\exp \left[-\left(\frac{t}{\mathrm{MDT}}\right)^{b}\right]\right)$

The equation to use was selected based on adequacy of fitting the in vitro dissolution data and the Akaike Information Criteria (AIC) from each equation [20].

1) Individual unit impulse response (UIR) parameters were generated using the IR formulation in vivo data. The individual UIR parameters were estimated by fitting a maximum of three polyexponential function with a time lag to each individual profile from the IR formulation and choosing the one which best fits the profile based on the AIC with a uniform weighting scheme. Since the reference formulation was an IR formulation, the "strip $\mathrm{K}_{\mathrm{a}}$ " approach was followed to ensure that the UIR is decoupled from the absorption process as previously described [21]. This assumes that absorption is truly first-order and fits a model that is ncompartment poly exponential. Within the model, the maximum number of UIR exponentials was set to 3. Additionally, a lag time $\left(T_{\text {lag }}\right)$ was incorporated into the model to allow for a time lag in absorption. The adequacy of the selected model was determined through evaluating the agreement between modelpredicted and observed upadacitinib plasma concentration versus time profiles.

2) The fraction of upadacitinib dose absorbed in vivo $\left(F_{\mathrm{a}}\right)$ from each of the four upadacitinib ER formulations relative to the IR formulation was estimated by numerical deconvolution of the observed plasma concentration versus time profiles against the UIR from the reference IR formulation of upadacitinib. Exploratory plots were generated to evaluate the relationship between $F_{\mathrm{a}}$ and $F_{\text {diss, vitro as well as }}$ between the in vitro dissolution time $\left(T_{\text {vitro }}\right)$ and in vivo absorption time $\left(T_{\text {vivo }}\right)$.

3) Several linear and non-linear models were evaluated for correlation between the fraction dissolved versus time and fraction absorbed versus time profiles of upadacitinib. Three formulations (formulation A, C, and D) were used to establish the IVIVC, and the fourth formulation (formulation B) was used for external validation. Default linear models in Phoenix IVIVC toolkit which employ absorption scale, time scale, and/or time shift for different dissolution conditions were initially evaluated. However, all the evaluated linear models demonstrated underprediction for upadacitinib $C_{\max }$ and a linear IVIVC could not be established. A user-specified non-linear IVIVC was evaluated by fitting a non-linear $E_{\max }$ model to scale the $T_{\text {vitro }}$ to the $T_{\text {vivo }}$, as shown in Eq. 2. After implementing non-linear time scaling, no additional scaling factor was used between $F_{\mathrm{a}}$ and $F_{\text {diss, vitro. }}$

$T_{\text {vitro }}=\left[\frac{\left(A 1 * T_{\text {vivo }}\right)}{\left(A 2+T_{\text {vivo }}\right)}\right]-B 2$

where $A 1, A 2$, and $B 2$ are constants characterizing the $E_{\max }$ relationship between $T_{\text {vitro }}$ and $T_{\text {vivo. }}$

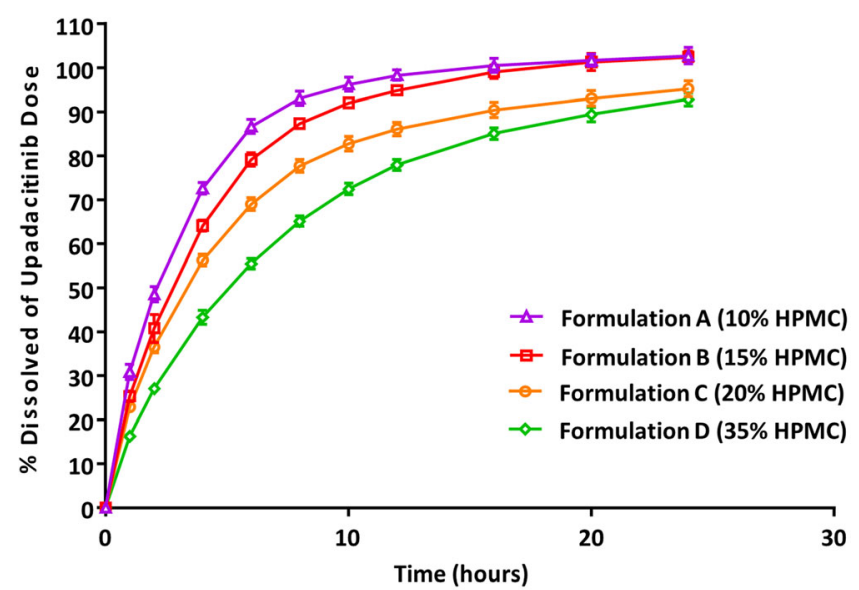

\begin{tabular}{|c|c|c|c|c|}
\hline f2 & A & B & C & D \\
\hline A & $\mathrm{n} / \mathrm{a}$ & & & \\
\hline B & 56.5 & $\mathrm{n} / \mathrm{a}$ & & \\
\hline C & 42.7 & 55.1 & $\mathrm{n} / \mathrm{a}$ & \\
\hline D & 31.1 & 37.0 & 49.0 & $\mathrm{n} / \mathrm{a}$ \\
\hline
\end{tabular}

Fig. 1. Cumulative percent dissolved $($ mean $\pm S D)$ versus time profiles for upadacitinib ER formulations containing 10\% HPMC (formulation A), 15\% HPMC (formulation B), 20\% HPMC (formulation C; target formulation), and 35\% HPMC (formulation D). F2 values comparing the four formulations are presented 


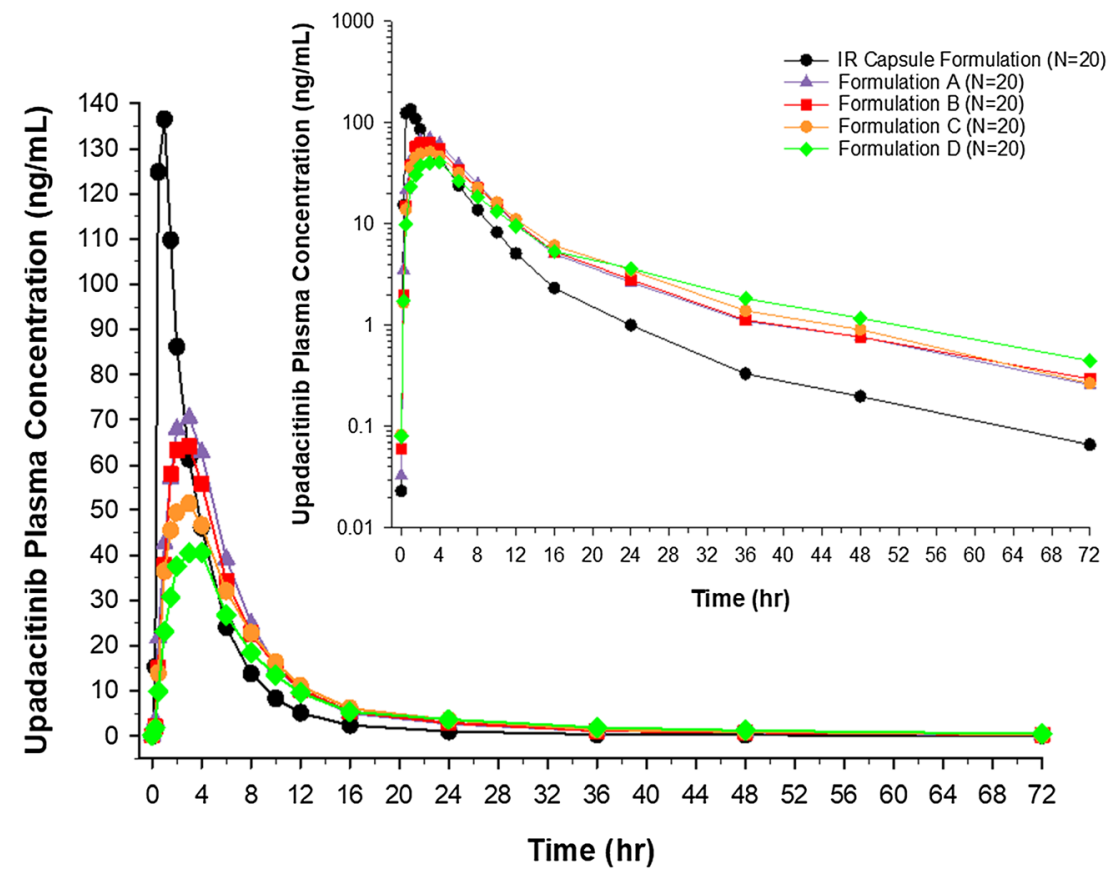

Fig. 2. Mean upadacitinib plasma concentration versus time profiles following administration of IR capsule and ER tablet formulations of upadacitinib. ER formulations of upadacitinib contained 10\% HPMC (formulation A), 15\% HPMC (formulation B), 20\% HPMC (formulation C; target formulation), and 35\% HPMC (formulation D). Insert: Loglinear scale of mean upadacitinib plasma concentration versus time profiles following administration of IR capsule and ER tablet formulations of upadacitinib

\section{Validation of the IVIVC}

The developed IVIVC relationship was used to predict $F_{\text {a }}\left(F_{\text {a, pred }}\right)$ corresponding to $F_{\text {diss, vitro }}$ at different time points for each formulation. The $F_{\mathrm{a}}$, pred for each of the four ER formulations and the individual UIRs from the IR capsule formulation were used as the input function for convolution

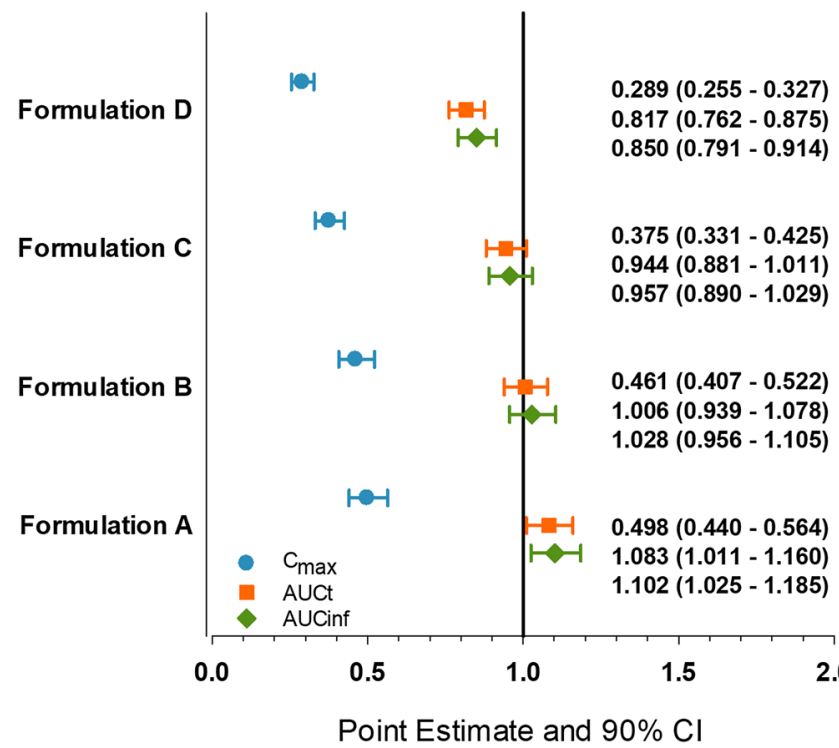

Fig. 3. Point Estimates and $90 \%$ confidence intervals for the bioavailability of upadacitinib following administration of single doses of upadacitinib $30 \mathrm{mg}$ extended-release tablets with different release rates relative to single $24 \mathrm{mg}$ dose of the upadacitinib immediaterelease capsules to generate individual predicted plasma concentration versus time profiles for the different ER formulations. The predicted pharmacokinetic parameters, $C_{\max }, \mathrm{AUC}_{\text {last }}$, and $\mathrm{AUC}_{\text {inf }}$ were estimated using non-compartmental analysis within the Phoenix IVIVC toolkit.

The prediction error, \% PE, was calculated for $\mathrm{AUC}_{\mathrm{inf}}$ and $C_{\max }$ for each ER formulation as:

[(predicted-observed)/observed $] \times 100 \%$

Additionally, a cross-validation was conducted using the leave-one-out approach. The final IVIVC model was re-run using each of the four extended-release formulations (A, B, $\mathrm{C}$, and $\mathrm{D})$ as an external validation formulation and the remaining three formulations for model building and internal validation. The \%PE was calculated for internal and external validation formulations for each of the cross-validation runs.

The predictive ability of the IVIVC was assessed for the numerical IVIVC through evaluating each method's internal and external predictability per FDA and EMA guidance documents $[17,18]$. To establish the internal predictability of the IVIVC, an average absolute \% PE of $10 \%$ or less for $C_{\text {max }}$ and $\mathrm{AUC}_{\text {inf }}$ was required. In addition, the absolute $\%$ $\mathrm{PE}$ for each individual formulation was required not to exceed $15 \%$. For external validation, the absolute \%PE was required not to exceed $10 \%$ for $C_{\max }$ and $\mathrm{AUC}_{\mathrm{inf}}$.

\section{RESULTS}

\section{In Vitro Dissolution Study}

The in vitro drug-release profiles for the four upadacitinib ER formulations using the selected method 
Table I. Pharmacokinetic Parameters of Upadacitinib Following Administration of Single Doses of Upadacitinib IR Capsules (24 mg) and ER Tablets $(30 \mathrm{mg}$ ) with Different Release Rates Under Fasting Conditions

\begin{tabular}{|c|c|c|c|c|c|}
\hline & & & Formulation & & \\
\hline $\begin{array}{l}\text { Pharmacokinetic } \\
\text { Parameters (units) }\end{array}$ & $\begin{array}{l}\text { IR Capsules } \\
\text { UPA } 24 \mathrm{mg} \\
(N=20)\end{array}$ & $\begin{array}{l}\text { ER formulation A } \\
10 \% \text { HPMC } \\
\text { UPA } 30 \mathrm{mg} \\
(N=20)\end{array}$ & $\begin{array}{l}\text { ER formulation B } \\
15 \% \text { HPMC } \\
\text { UPA } 30 \mathrm{mg} \\
(N=20)\end{array}$ & $\begin{array}{l}\text { ER formulation } C^{*} \\
20 \% \text { HPMC } \\
\text { UPA } 30 \mathrm{mg} \\
(N=20)\end{array}$ & $\begin{array}{l}\text { ER formulation D } \\
35 \% \text { HPMC } \\
\text { UPA } 30 \mathrm{mg} \\
(N=20)\end{array}$ \\
\hline $\begin{array}{l}C_{\max }(\mathrm{ng} / \mathrm{mL}) \\
T_{\max }{ }^{a}(\mathrm{~h}) \\
\operatorname{AUC}_{\mathrm{t}}(\mathrm{ng} \cdot \mathrm{h} / \mathrm{mL}) \\
\mathrm{AUC}_{\mathrm{inf}}(\mathrm{ng} \cdot \mathrm{h} / \mathrm{mL}) \\
\mathrm{t}_{1 / 2}{ }^{\mathrm{b}}(\mathrm{h})\end{array}$ & $\begin{array}{l}159 \pm 45.7 \\
1.0(0.5-2.0) \\
507 \pm 85.0 \\
510 \pm 85.1 \\
10.2(6.92)\end{array}$ & $\begin{array}{l}79.3 \pm 24.3 \\
2.0(0.5-4.0) \\
549 \pm 89.5 \\
562 \pm 89.3 \\
12.2(8.24)\end{array}$ & $\begin{array}{l}72.6 \pm 19.7 \\
2.5(1.5-4.0) \\
515 \pm 114 \\
529 \pm 111 \\
10.7(7.65)\end{array}$ & $\begin{array}{l}59.5 \pm 16.7 \\
3.0(1.0-6.0) \\
487 \pm 120 \\
497 \pm 121 \\
9.96(6.09)\end{array}$ & $\begin{array}{l}46.2 \pm 14.7 \\
3.0(1.0-6.0) \\
422 \pm 106 \\
443 \pm 113 \\
12.5(8.06)\end{array}$ \\
\hline
\end{tabular}

UPA upadacitinib

*Target formulation

${ }^{a}$ Median (minimum through maximum)

${ }^{b}$ Harmonic mean (pseudo-standard deviation)

(USP App 1 at $\mathrm{pH}$ 6.8) are shown in Fig. 1 along with f2 values comparing the different formulations to each other. Formulations A, B, C, and D released at least $80 \%$ of drug by $6,8,10$, and $16 \mathrm{~h}$, respectively. To evaluate the possibility of linear IVIVC, additional dissolution conditions were evaluated, but a linear IVIVC that could meet the FDA required predictions error criteria was not established (Supplemental Table 1). A Weibull function (Eq. 2) was used to fit the in vitro dissolution data as it provided better fit compared to a Hill function (Eq. 1) and resulted in lower AIC values by 10 to 35 points (for formulations $\mathrm{A}, \mathrm{B}, \mathrm{C}$, and $\mathrm{D}$, AIC values were $-61,-63,-62$, and -84 , respectively, for Weibull function and $-40,-53,-39$, and -48 , respectively, for the Hill function).

\section{In Vivo Bioavailability Study Results}

The mean plasma concentration versus time profiles from the in vivo study for the ER formulations A, B, C, and $\mathrm{D}$ and the IR formulations are presented in Fig. 2. The evaluated ER formulations (A, B, C, and D) showed central ratios of upadacitinib $\mathrm{AUC}_{\text {inf }}$ and $C_{\max }$ relative to the reference IR capsule formulation in the rank order of their in vitro release rate and HPMC content (Fig. 3). The ratios of central values for upadacitinib $C_{\max }$ were $0.5,0.46,0.38$, and
0.29 for formulations $\mathrm{A}, \mathrm{B}, \mathrm{C}$, and $\mathrm{D}$, respectively, relative to the IR capsule formulation. The ratios of the central values for upadacitinib $\mathrm{AUC}_{\mathrm{inf}}$ were 1.1, 1.0, 0.96, and 0.85 for formulations $\mathrm{A}, \mathrm{B}, \mathrm{C}$, and $\mathrm{D}$, respectively, relative to the IR capsule formulation. The pharmacokinetic parameters of upadacitinib following administration of single doses of upadacitinib IR capsules (24 mg) and ER tablets (30 mg) are described in Table I. Median $T_{\max }$ of upadacitinib was 2.0, 2.5, 3.0, and $3.0 \mathrm{~h}$ for ER formulations A, B, C, and D, respectively, as compared to $1.0 \mathrm{~h}$ for the IR formulation. Upadacitinib terminal half-life was similar across the ER formulations and comparable to that of the IR formulation (approximately 10 to $13 \mathrm{~h}$ ).

\section{IVIVC Model}

The mean fraction of upadacitinib dose absorbed versus time profiles are presented in Fig. 4 for the different extended-release formulations based on deconvolution. Exploratory plots for the fraction of upadacitinib $F_{\mathrm{a}}$ and $F_{\mathrm{diss}}$, vitro as well as between $T_{\text {vivo }}$ and $T_{\text {vitro }}$ are presented in Fig. 5. Based on the exploratory plots, the relation between $F_{\mathrm{a}}$, obs

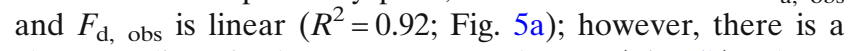
clear non-linearity between $T_{\text {vivo }}$ and $T_{\text {vitro }}$ (Fig. $5 \mathrm{~b}$ ). Therefore, default linear models with Phoenix IVIVC toolkit could

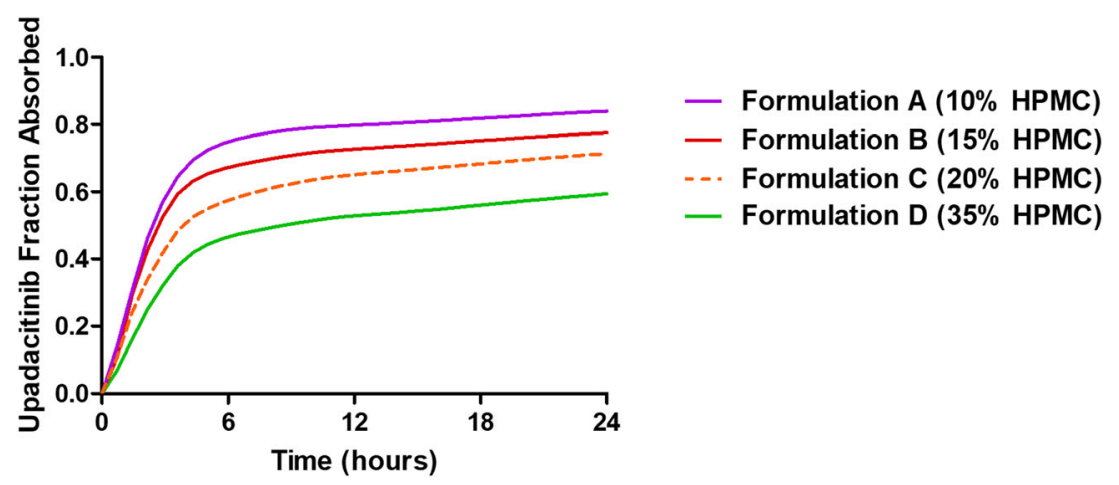

Fig. 4. Mean in vivo absorption versus time profile of upadacitinib extended-release formulations based on numerical deconvolution 
a

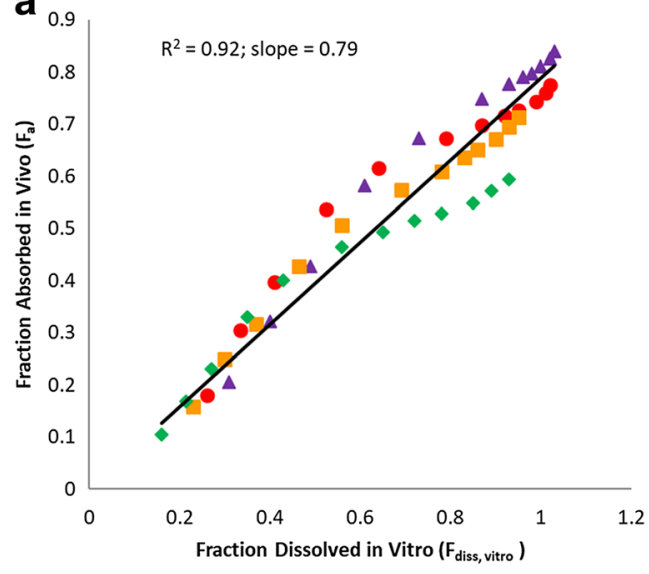

b

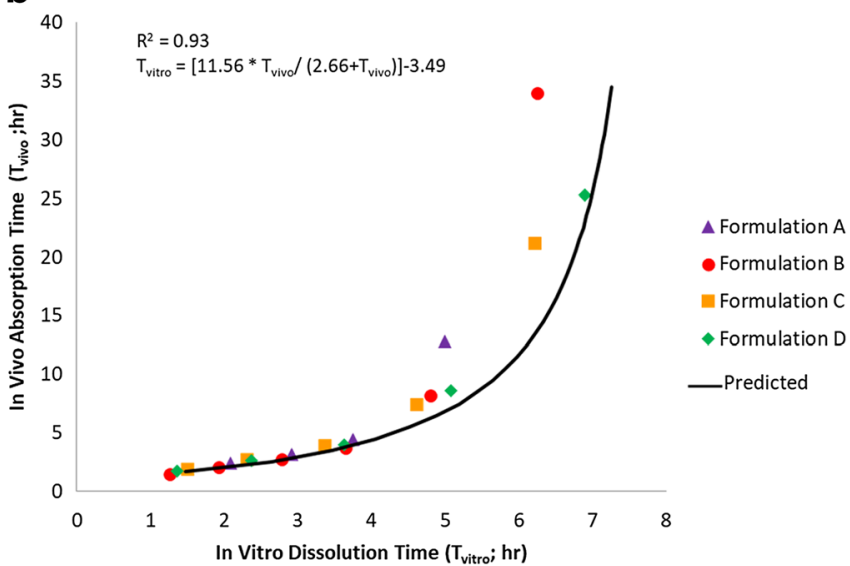

Fig. 5. Correlation between a the observed fraction absorbed and the fraction dissolved and $\mathbf{b}$ in vivo absorption time and in vitro dissolution time for upadacitinib ER formulations

not be used to establish an IVIVC for upadacitinib ER formulations. The relationship between $T_{\text {vitro }}$ and time $T_{\text {vivo }}$ was best described by a non-linear $E_{\max }$ function with intercept (Eq. 2) as described in methods and presented in Fig. 5b. The mean estimates (and standard error of the estimates) of the IVIVC parameters (Eq. 2) were 11.6 (0.42) h for A1, $2.66(0.28) \mathrm{h}$ for A2, and $3.49(0.47) \mathrm{h}$ for B2.

\section{Internal and External Validation}

Summary of the observed and predicted upadacitinib $\mathrm{AUC}_{\text {inf }}$ and $\mathrm{C}_{\max }$ for each of the three formulations used to develop the IVIVC (formulations A, C, and D) as well as for the external validation formulation (formulation $\mathrm{B}$ ) are presented in Table II. The average \% PE was less than $10 \%$ for both $\mathrm{AUC}_{\text {inf }}$ and $C_{\max }$ for the ER formulations $(\mathrm{A}, \mathrm{C}$, and D). The \%PE for each of the individual ER formulations A, $\mathrm{C}$, and $\mathrm{D}$ were also well below $15 \%$ for both $\mathrm{AUC}_{\mathrm{inf}}$ and $C_{\text {max }}$. In addition, the external \% PE for ER formulation B was less than $10 \%$ (Table II). These results demonstrate that the established IVIVC meets the predefined internal and external validation criteria.

Results from the cross-validation using the leave-one-out approach are presented in Table III. All cross-validation runs met the acceptance criteria (\% PE for each formulation < $15 \%$; average internal validation $\% \mathrm{PE}<10 \%$; external validation $\% \mathrm{PE}<10 \%)$.

\section{DISCUSSION}

A predictive level A non-linear IVIVC was established for upadacitinib ER formulation using in vivo and in vitro data for formulations containing a range of the releasecontrolling polymer (HPMC) of 10 to $35 \%$. This range encompasses the target to be marketed formulation (formulation C) which contains 20\% HPMC. The established IVIVC meets both the internal and external predictability per the FDA and EMA criteria and can potentially be used as surrogate for in vivo study if there is a need for formulation change (within the design space of the IVIVC) or manufacturing process change. Additionally, the established IVIVC enables setting the release specifications based on clinical relevance using the predicted range of upadacitinib exposures for formulations that fall within the proposed specifications. These analyses highlight the importance of evaluating non-linear models when linear IVIVC relationships cannot be established.

Table II. Results of the Internal and External Validation for Upadacitinib IVIVC

\begin{tabular}{|c|c|c|c|c|c|c|c|c|c|c|c|c|c|c|c|}
\hline \multicolumn{13}{|c|}{ Internal validation formulations } & \multirow{2}{*}{\multicolumn{3}{|c|}{$\begin{array}{l}\text { External validation } \\
\text { formulation } \\
\text { Formulation B } \\
15 \% \text { HPMC }\end{array}$}} \\
\hline \multirow[b]{2}{*}{ Parameter } & \multicolumn{3}{|c|}{$\begin{array}{l}\text { Formulation A } \\
10 \% \text { HPMC }\end{array}$} & \multicolumn{3}{|c|}{$\begin{array}{l}\text { Formulation C } \\
\text { (target formulation) } \\
20 \% \text { HPMC }\end{array}$} & \multicolumn{3}{|c|}{$\begin{array}{l}\text { Formulation D } \\
35 \% \text { HPMC }\end{array}$} & \multicolumn{3}{|c|}{$\begin{array}{l}\text { Average for } \\
\text { internal validation }\end{array}$} & & & \\
\hline & Obs. & Pred. & $\% \mathrm{PE}$ & Obs. & Pred. & $\% \mathrm{PE}$ & Obs. & Pred. & $\% \mathrm{PE}$ & Obs. & Pred. & Absolute \% PE & Obs. & Pred. & $\% \mathrm{PE}$ \\
\hline$C_{\max }(\mathrm{ng} / \mathrm{mL})$ & 75.9 & 78.6 & 3.55 & 57.2 & 58.0 & 1.40 & 44.0 & 44.6 & 1.22 & 57.6 & 58.8 & 2.06 & 70.2 & 68.2 & -2.85 \\
\hline $\operatorname{AUC}_{\text {last }}(\mathrm{h} \cdot \mathrm{ng} / \mathrm{mL})$ & 532.0 & 561.8 & 5.60 & 463.2 & 462.6 & -0.13 & 400.5 & 389.1 & -2.84 & 462.1 & 465.9 & 2.86 & 494.1 & 524.1 & 6.08 \\
\hline $\mathrm{AUC}_{\text {inf }}(\mathrm{h} \cdot \mathrm{ng} / \mathrm{mL})$ & 545.1 & 565.3 & 3.70 & 472.9 & 466.9 & -1.27 & 420.0 & 393.8 & -6.24 & 476.6 & 470.2 & 3.74 & 508.2 & 528.3 & 4.00 \\
\hline
\end{tabular}

Note: Values are reported as the geometric mean 
Table III. Cross-Validation Results for the Non-Linear IVIVC Model Using the Leave-One-Out Approach

\begin{tabular}{|c|c|c|c|c|c|}
\hline $\begin{array}{l}\text { Validation } \\
\text { Initial correlation estimates } \\
\text { Final parameter estimates }\end{array}$ & Formulation & Parameter $^{\mathrm{a}}$ & Predicted & Observed & $\% \mathrm{PE}$ \\
\hline $\begin{array}{l}\text { Model developed with formulations A, C, and D. } \\
\text { Formulation B used for external validation } \\
\text { Initial estimates: } \\
\text { A1: } 15 \mathrm{~h} \text {; A2: } 5 \text { h; B2: } 8 \mathrm{~h} \\
\text { Final Estimates: } \\
\text { A1: } 11.6 \mathrm{~h} \text {; A2: } 2.7 \mathrm{~h} \text {; B2: } 3.5 \mathrm{~h}\end{array}$ & $\begin{array}{l}\text { A } \\
\text { A } \\
\text { C } \\
\text { C } \\
\text { D } \\
\text { D } \\
\text { Avg internal } \\
\text { Avg internal } \\
\text { B } \\
\text { B }\end{array}$ & $\begin{array}{l}\mathrm{AUC}_{\mathrm{inf}} \\
C_{\mathrm{max}} \\
\mathrm{AUC}_{\mathrm{inf}} \\
C_{\max } \\
\mathrm{AUC}_{\mathrm{inf}} \\
C_{\max } \\
\mathrm{AUC}_{\mathrm{inf}} \\
C_{\max } \\
\mathrm{AUC} \mathrm{C}_{\mathrm{inf}} \\
C_{\max }\end{array}$ & $\begin{array}{r}565 \\
79 \\
467 \\
58 \\
394 \\
45 \\
470 \\
59 \\
528 \\
68\end{array}$ & $\begin{array}{r}545 \\
76 \\
473 \\
57 \\
420 \\
44 \\
477 \\
58 \\
508 \\
70\end{array}$ & $\begin{array}{r}3.7 \\
3.6 \\
-1.3 \\
1.4 \\
-6.2 \\
1.2 \\
3.7 \\
2.1 \\
4.0 \\
-2.9\end{array}$ \\
\hline $\begin{array}{l}\text { Model developed with formulations B, C, and D. } \\
\text { Formulation A used for external validation } \\
\text { Initial estimates: } \\
\text { A1: } 15 \text { h; A2: } 5 \mathrm{~h} \text {; B2: } 8 \mathrm{~h} \\
\text { Final estimates: } \\
\text { A1: } 11.6 \mathrm{~h} \text {; A2: } 2.4 \mathrm{~h} \text {; B2: } 3.7 \mathrm{~h}\end{array}$ & $\begin{array}{l}\text { B } \\
\text { B } \\
\text { C } \\
\text { C } \\
\text { D } \\
\text { D } \\
\text { Avg internal } \\
\text { Avg internal } \\
\text { A } \\
\text { A }\end{array}$ & $\begin{array}{l}\mathrm{AUC}_{\mathrm{inf}} \\
C_{\max } \\
\mathrm{AUC}_{\mathrm{inf}} \\
C_{\max } \\
\mathrm{AUC} C_{\mathrm{inf}} \\
C_{\max } \\
\mathrm{AUC} C_{\mathrm{inf}} \\
C_{\max } \\
\mathrm{AUC} C_{\mathrm{inf}} \\
C_{\max }\end{array}$ & $\begin{array}{r}527 \\
69 \\
465 \\
59 \\
392 \\
45 \\
458 \\
57 \\
565 \\
79\end{array}$ & $\begin{array}{r}508 \\
70 \\
473 \\
57 \\
420 \\
44 \\
466 \\
56 \\
545 \\
76\end{array}$ & $\begin{array}{r}3.7 \\
-1.9 \\
-1.6 \\
2.4 \\
-6.7 \\
2.5 \\
4.0 \\
2.2 \\
3.6 \\
4.4\end{array}$ \\
\hline $\begin{array}{l}\text { Model developed with formulations A, B, and C. } \\
\text { Formulation D used for external validation } \\
\text { Initial estimates: } \\
\text { A1: } 16 \text { h; A2: } 6 \mathrm{~h} \text {; B2: } 10 \mathrm{~h} \\
\text { Final estimates: } \\
\text { A1: } 17.1 \mathrm{~h} \text {; A2: } 3.2 \mathrm{~h} \text {; B2: } 7.3 \mathrm{~h}\end{array}$ & $\begin{array}{l}\text { A } \\
\text { A } \\
\text { B } \\
\text { B } \\
\text { C } \\
\text { C } \\
\text { Avg internal } \\
\text { Avg internal } \\
\text { D } \\
\text { D }\end{array}$ & $\begin{array}{l}\mathrm{AUC}_{\mathrm{inf}} \\
C_{\max } \\
\mathrm{AUC}_{\mathrm{inf}} \\
C_{\max } \\
\mathrm{AUC}_{\mathrm{inf}} \\
C_{\max } \\
\mathrm{AUC} C_{\mathrm{inf}} \\
C_{\max } \\
\mathrm{AUC} C_{\mathrm{inf}} \\
C_{\max }\end{array}$ & $\begin{array}{r}592 \\
73 \\
561 \\
63 \\
503 \\
55 \\
551 \\
63 \\
433 \\
41\end{array}$ & $\begin{array}{r}545 \\
76 \\
508 \\
70 \\
473 \\
57 \\
508 \\
67 \\
420 \\
44\end{array}$ & $\begin{array}{r}8.6 \\
-3.6 \\
10.4 \\
-10.2 \\
6.4 \\
-4.4 \\
8.4 \\
6.0 \\
3.1 \\
-6.3\end{array}$ \\
\hline $\begin{array}{l}\text { Model developed with formulations A, B, and D. } \\
\text { Formulation C used for external validation } \\
\text { Initial estimates: } \\
\text { A1: } 16 \text { h; A2: } 6 \text { h; B2: } 10 \mathrm{~h} \\
\text { Final estimates: } \\
\text { A1: } 11.6 \mathrm{~h} \text {; A2: } 2.0 \mathrm{~h} \text {; B2: } 4.2 \mathrm{~h}\end{array}$ & $\begin{array}{l}\text { A } \\
\text { A } \\
\text { B } \\
\text { B } \\
\text { D } \\
\text { D } \\
\text { Avg internal } \\
\text { Avg internal } \\
\text { C } \\
\text { C }\end{array}$ & $\begin{array}{l}\mathrm{AUC}_{\mathrm{inf}} \\
C_{\max } \\
\mathrm{AUC}_{\mathrm{inf}} \\
C_{\max } \\
\mathrm{AUC}_{\mathrm{inf}} \\
C_{\max } \\
\mathrm{AUC} C_{\mathrm{inf}} \\
C_{\max } \\
\mathrm{AUC} C_{\mathrm{inf}} \\
C_{\max }\end{array}$ & $\begin{array}{r}558 \\
79 \\
518 \\
69 \\
380 \\
45 \\
479 \\
63 \\
455 \\
59\end{array}$ & $\begin{array}{r}545 \\
76 \\
508 \\
70 \\
420 \\
44 \\
488 \\
62 \\
473 \\
57\end{array}$ & $\begin{array}{r}2.4 \\
4.4 \\
1.8 \\
-1.6 \\
-9.6 \\
2.9 \\
4.6 \\
3.0 \\
-3.8 \\
2.6\end{array}$ \\
\hline
\end{tabular}

${ }^{a}$ Units are $\mathrm{ng} \cdot \mathrm{h} / \mathrm{mL}$ for $\mathrm{AUC}_{\mathrm{inf}}$ and $\mathrm{ng} / \mathrm{mL}$ for $C_{\max }$

Non-linear IVIVC models, although less common than linear models, are considered acceptable as long as internal and/or external validation demonstrates adequate predictability of the IVIVC and that the same non-linear time scale is used for all formulations [17-19]. Evaluation of linear models for upadacitinib ER formulation consistently resulted in under-prediction of upadacitinib $C_{\max }$, (data not shown) suggesting slower dissolution in vitro than in vivo dissolution that was not adequately accounted for using a linear time scaling factor. This was also the case for various other in vitro test conditions evaluated to mimic the in vivo conditions
(Supplemental Table 1). In general, potential causes for nonlinearity in IVIVC can be expected to be the lack of uniformity in absorption of a drug throughout the gastrointestinal tract, possible saturation of transporters or first-pass metabolic pathways, or the nature of the formulation [22,23].

Upadacitinib is a highly soluble and highly permeable drug that exhibits linear pharmacokinetic characteristics over the range of doses that have been studied (up to a dose of $45 \mathrm{mg}$ using the extended-release formulation and up to $48 \mathrm{mg}$ using the immediate-release formulation). Given the high permeability and high solubility of upadacitinib, 
intestinal transporters are not expected to have clinically relevant role in upadacitinib disposition in vivo and no data suggest lack of uniformity in absorption throughout the gastrointestinal tract in humans. Therefore, it is likely that the nature of the formulation and how it releases the drug in vivo is driving the non-linear relationship between $T_{\text {vitro }}$ and $T_{\text {vivo. Analysis of deconvolved in vivo absorption profiles }}$ (Fig. 4) demonstrated a common two-stage profile for all four tested formulations: rapid early absorption over the first 4 to $5 \mathrm{~h}$, followed by slower absorption afterward. Drug release from gel-forming hydrophilic matrices (such as that used in this upadacitinib controlled release formulation) has been known to come from two mechanisms: diffusion of drug molecules across the gel layer formed on the surface of the tablet and erosion of the polymer on the tablet surface (or the outer layer of the gel). The dissolution conditions tested in vitro (including those that used two different $\mathrm{pHs}$ or RPMs in the same test; Supplemental Table 1) were unable to mimic the time course of the release in vivo for all formulations; thus, establishing a linear IVIVC relationship was not feasible. Only a non-linear IVIVC with prediction errors meeting the acceptance criteria could be achieved no matter what dissolution condition was used. An important consideration for the development and validation of this non-linear correlation is that the model fitted to correct for the time scale difference between the in vitro data and the in vivo data is the same for all the formulations tested and is independent of the formulation or its release rate.

It is worth noting that the relationship between $T_{\text {vitro }}$ and $T_{\text {vivo }}$ appeared linear up to approximately $8 \mathrm{~h}$ in vivo. An approach that is alternative to the use of non-linear model would be to establish a linear IVIVC with in vivo cutoff time ( $\left.t_{\text {cutoff }}\right)$. However, use of $t_{\text {cutoff }}$ implies ignoring collected in vivo and in vitro data after the selected $t_{\text {cutoff. Additionally, }}$ Phoenix software that does not allow estimating the optimal $t_{\text {cutoff }}$ value and $t_{\text {cutoff }}$ will need to be selected as an arbitrary value based on observed data. Both of the aforementioned points can potentially introduce bias in the analysis when $t_{\text {cutoff }}$ is used. We opted to use a non-linear IVIVC approach rather than use of linear IVIVC with $t_{\text {cutoff }}$ to be able to use the totality of the data thus to avoid ignoring any in vitro or in vivo data and to allow the software to estimate the bestfitting parameters for the non-linear relationship based on the data.

The IVIVC model was developed while a Phase 3 study which evaluated the safety and efficacy of upadacitinib doses of $15 \mathrm{mg}$ and $30 \mathrm{mg}$ QD using the ER formulation was ongoing. The upadacitinib ER formulation is considered proportionally similar between the 15 and $30 \mathrm{mg}$ strengths, and upadacitinib plasma exposures are linear over a wide range of IR and ER doses [8,15]. Therefore, the IVIVC, developed using the highest clinically relevant strength at the time of conducting the analysis (30 mg strength), is considered applicable to the $15 \mathrm{mg}$ strength.

The robustness of the model was evaluated through cross-validation using the leave-one-out approach; all crossvalidation runs meet the acceptance criteria (\%PE for each formulation $<15 \%$; average internal validation $\% \mathrm{PE}<10 \%$; external validation \% PE $<10 \%$ ). This assessment, although not a requirement per regulatory guidance documents, demonstrate robustness of the IVIVC and that it is not sensitive to data from a specific formulation. With a validated IVIVC, an in vitro test can potentially serve as a surrogate for bioavailability testing as well as a tool to screen formulations and set the dissolution and drug-release acceptance criteria.

\section{CONCLUSION}

A robust non-linear level A correlation that meets the FDA and EMA validation criteria for both internal and external predictability was established for upadacitinib ER formulation. This IVIVC can be used as surrogate for bioequivalence studies in case of future formulation changes that are covered by the IVIVC release rates tested. This correlation will enable the setting of clinically meaningful dissolution specifications based on acceptable differences in plasma concentrations corresponding to the upper and lower limit of the dissolution specifications.

\section{ACKNOWLEDGEMENTS}

This study was sponsored by AbbVie Inc. AbbVie Inc. contributed to the study design; research; data interpretation; and writing, review, and approval of the manuscript for publication. Medical writing support was provided by Wesley Wayman, an employee of AbbVie Inc.

\section{COMPLIANCE WITH ETHICAL STANDARDS}

Conflict of Interest All authors are employees of AbbVie Inc. and may hold AbbVie stocks or options.

Data Sharing Statement AbbVie is committed to responsible data sharing regarding the clinical trials we sponsor. This includes access to anonymized, individual and trial-level data (analysis data sets), and other information (e.g., protocols and Clinical Study Reports), as long as the trials are not part of an ongoing or planned regulatory submission. This includes requests for clinical trial data for unlicensed products and indications.

This clinical trial data can be requested by any qualified researchers who engage in rigorous, independent scientific research and will be provided following review and approval of a research proposal and Statistical Analysis Plan (SAP) and execution of a Data Sharing Agreement (DSA). Data requests can be submitted at any time and the data will be accessible for 12 months, with possible extensions considered. For more information on the process, or to submit a request, visit the following link: https://www.abbvie.com/our-science/ clinical-trials/clinical-trials-data-and-information-sharing/ data-and-information-sharing-with-qualified-researchers.html

Open Access This article is distributed under the terms of the Creative Commons Attribution 4.0 International License (http://creativecommons.org/licenses/by/4.0/), which permits unrestricted use, distribution, and reproduction in any medium, provided you give appropriate credit to the original author(s) and the source, provide a link to the Creative Commons license, and indicate if changes were made. 


\section{REFERENCES}

1. Parmentier JM, Voss J, Graff C, Schwartz A, Argiriadi M, Friedman $\mathrm{M}$, et al. In vitro and in vivo characterization of the JAK1 selectivity of upadacitinib (ABT-494). BMC Rheumatol. 2018;2(1):23. https://doi.org/10.1186/s41927-018-0031-x.

2. Burmester GR, Kremer JM, Van den Bosch F, Kivitz A, Bessette L, Li Y, et al. Safety and efficacy of upadacitinib in patients with rheumatoid arthritis and inadequate response to conventional synthetic disease-modifying anti-rheumatic drugs (SELECT-NEXT): a randomised, double-blind, placebocontrolled phase 3 trial. Lancet. 2018;391(10139):2503-12. https://doi.org/10.1016/S0140-6736(18)31115-2.

3. Leonard WJ. Role of Jak kinases and STATs in cytokine signal transduction. Int J Hematol. 2001;73(3):271-7.

4. O'Shea JJ, Plenge R. JAK and STAT signaling molecules in immunoregulation and immune-mediated disease. Immunity. 2012;36(4):542-50. https://doi.org/10.1016/j.immuni.2012.03.014.

5. Kremer JM, Emery P, Camp HS, Friedman A, Wang L, Othman AA, et al. A phase IIb study of ABT-494, a selective JAK-1 inhibitor, in patients with rheumatoid arthritis and an inadequate response to anti-tumor necrosis factor therapy. Arthritis Rheum. 2016;68(12):2867-77. https://doi.org/10.1002/art.39801.

6. Genovese MC, Smolen JS, Weinblatt ME, Burmester GR, Meerwein S, Camp HS, et al. Efficacy and safety of ABT-494, a selective JAK-1 inhibitor, in a phase IIb study in patients with rheumatoid arthritis and an inadequate response to methotrexate. Arthritis Rheumatol. 2016;68(12):2857-66. https://doi.org/ 10.1002/art.39808.

7. Genovese MC, Fleischmann R, Combe B, Hall S, Rubbert-Roth A, Zhang Y, et al. Safety and efficacy of upadacitinib in patients with active rheumatoid arthritis refractory to biologic diseasemodifying anti-rheumatic drugs (SELECT-BEYOND): a double-blind, randomised controlled phase 3 trial. Lancet. 2018;391(10139):2513-24. https://doi.org/10.1016/S01406736(18)31116-4.

8. Mohamed MF, Zeng J, Marroum PJ, Song IH, Othman AA. Pharmacokinetics of upadacitinib with the clinical regimens of the extended-release formulation utilized in rheumatoid arthritis phase 3 trials. Clin Pharmacol Drug Dev. 2019;8(2):208-16. https://doi.org/10.1002/cpdd.462.

9. Fleischmann R, Pangan AL, Song IH, Mysler E, Bessette L, Peterfy C, et al. Upadacitinib versus Placebo or Adalimumab in Patients with Rheumatoid Arthritis and an Inadequate Response to Methotrexate: Results of a Phase 3, Double-Blind, Randomized Controlled Trial. Arthritis Rheumatol. 2019. https://doi.org/10.1002/art.41032.

10. Van Vollenhoven R, Takeuchi T, Pangan AL, Friedman A, Mohamed MF, Chen S, et al. A phase 3, randomized, controlled trial comparing upadacitinib monotherapy to MTX monotherapy in MTX-naive patients with active rheumatoid arthritis [abstract]. Arthritis and Rheumatol. 2018;70(suppl 10).

11. Mohamed MF, Camp HS, Jiang P, Padley RJ, Asatryan A, Othman AA. Pharmacokinetics, safety and tolerability of ABT494, a novel selective JAK 1 inhibitor, in healthy volunteers and subjects with rheumatoid arthritis. Clin Pharmacokinet. 2016;55(12):1547-58. https://doi.org/10.1007/s40262-016-0419-y.

12. Mohamed MF, Zeng J, Marroum PJ, Song IH, \& Othman AA. Pharmacokinetics of upadacitinib with the clinical regimens of the extended-release formulation utilized in rheumatoid arthritis phase 3 trials. Clin Pharmacol Drug Dev. 2019;8(2):208-216. https://doi.org/10.1002/cpdd.462.

13. Klunder B, Mohamed MF, Othman AA. Population pharmacokinetics of upadacitinib in healthy subjects and subjects with rheumatoid arthritis: analyses of phase I and II clinical trials. Clin Pharmacokinet. 2018;57(8):977-88. https://doi.org/10.1007/ s40262-017-0605-6.

14. Nader A, Stodtmann S, Friedel A, Mohamed MF, \& Othman AA. Pharmacokinetics of upadacitinib in healthy subjects and subjects with rheumatoid arthritis, Crohn's disease, ulcerative colitis or atopic dermatitis: population analyses of Phase 1 and 2 clinical trials. 2019; In Press.

15. Klünder B, Mittapalli RK, Mohamed M-EF, Friedel A, Noertersheuser P, Othman AA. Population pharmacokinetics of upadacitinib using the immediate-release and extendedrelease formulations in healthy subjects and subjects with rheumatoid arthritis: analyses of phase I-III clinical trials. Clin Pharmacokinet. 2019;58:1045-58. https://doi.org/10.1007/s40262019-00739-3.

16. FDA. Guidance for Industry. Waiver of in vivo bioavailability and bioequivalence studies for immediate-release solid oral dosage forms based on a biopharmaceutics classification system. h t t p s://ww w.fda.gov/downloads/Drugs / GuidanceComplianceRegulatoryInformation/Guidances/ UCM070246.pdf Accessed January 24 2019. 2017.

17. FDA. Guidance for Industry. Extended release oral dosage forms: development, evaluation, and application of in vitro/in vivo correlations. US Department of Health and Human Services, Food and Drug Administration, Center for Drug Evaluation and Research (CDER). Available from: https:// www.fda.gov/downloads/drugs/guidances/ucm070239.pdf Accessed January 10 2019. 1997.

18. EMA. European Medicines Agency. Guidance on the Investigation of Bioequivalence. 2010. Available from: https:// www.ema.europa.eu/en/documents/scientific-guideline/guideline-investigation-bioequivalence-rev1_en.pdf. Accessed September 162019.

19. Suarez-Sharp S, Li M, Duan J, Shah H, Seo P. Regulatory experience with in vivo in vitro correlations (IVIVC) in new drug applications. AAPS J. 2016;18(6):1379-90. https://doi.org/ 10.1208/s12248-016-9966-2.

20. Sakamoto Y, Ishiguro M, \& Kitagawa G (1986) Akaike information criterion statistics. KTK Scientific Publishers ; D. Reidel ; Sold and distributed in the U.S.A. and Canada by Kluwer Academic Publishers: Tokyo; Dordrecht; Boston; Hingham, MA.

21. Kakhi M, Marroum P, Chittenden J. Analysis of level A in vitroin vivo correlations for an extended-release formulation with limited bioavailability. Biopharm Drug Dispos. 2013;34(5):26277. https://doi.org/10.1002/bdd.1820.

22. Sirisuth N, Augsburger LL, Eddington ND. Development and validation of a non-linear IVIVC model for a diltiazem extended release formulation. Biopharm Drug Dispos. 2002;23(1):1-8.

23. Cardot JM, Davit BM. In vitro-in vivo correlations: tricks and traps. AAPS J. 2012;14(3):491-9. https://doi.org/10.1208/s12248012-9359-0.

Publisher's Note Springer Nature remains neutral with regard to jurisdictional claims in published maps and institutional affiliations. 